\title{
Wie viel Staat braucht das Gesundheitswesen?
}

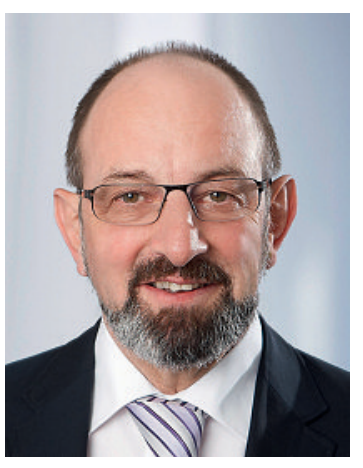

In letzter Zeit mehren sich die Ansprüche, dem Bund mehr Entscheidungsmöglichkeiten im Gesundheitswesen zu übertragen und damit die bisher favorisierten Verhandlungslösungen zwischen den Tarifpartnern abzulösen. In der letzten Woche haben die Gesundheitskommissionen von National- und Ständerat beschlossen, bei der TARMEDTarifstruktur dem Bundesrat subsidiär die Möglichkeit zu geben einzugreifen. Ein klarer Entscheid, der in Richtung eines Amtstarifs geht.

\section{Amtstarife führen nicht zum Ziel.}

Problematisch ist, dass Kostenträger, insbesondere santésuisse, aber auch Parlamentarier und die Eidgenössische Finanzkontrolle (EFK) bei der Evaluation der Tarifstruktur TARMED immer wieder die Eingriffe an der Tarifstruktur und die Tarifierung von Leistungen vermischen. Den Positionen in der Tarifstruktur sind eine standardisierte Erfassung von Minutagen sowie Kosten, und sie sind mit einer qualitativen Gewichtung der Leistungen hinterlegt. Sind die Positionen einmal in die Tarifstruktur aufgenommen, bilden sie die korrekten Verhältnisse der Leistungen untereinander ab. Dann erst erfolgt in einem zweiten Schritt die Tarifierung über die Taxpunktwerte. Während die Tarifstruktur gemäss der Vorgabe des KVG national einheitlich zu sein hat und der Genehmigung des Bundesrates untersteht, liegt die Genehmigung des KVG-Tarifs in Form des Tarifvertrages in der Kompetenz und Hoheit der Kantone. Die Genehmigungsbehörden für KVG-Tarifverträge sind die Kantonsregierungen.

Die Gesundheitsdirektorenkonferenz (GDK) und die EFK wollen den TARMED als Amtstarif in die Kompetenz und Verantwortung des Bundes und damit ins BAG zu überführen, was die kantonale Tarifhoheit untergräbt.

Das BAG zeigt in seinem Beibericht zum EFK-Bericht auch deutlich auf, dass es als Bundesamt für die Ausgestaltung eines Amtstarifs weder über die nötigen Ressourcen und Kompetenzen verfügt, noch gesetzliche Grundlagen für einen solchen Schritt bestehen.

$\mathrm{Zu}$ welchen unabsehbaren und ungewollten Folgen solche Bundeseingriffe führen können, zeigt die Revision der Analysenliste: $20 \%$ Absenkung bei den frei praktizierenden Ärz- ten - bei den Grundversorgern gar 30\% - war ebenso wenig vorgesehen wie der Kostenanstieg bei den Privatlabors!

Die Forderung, die ärztliche Medikamentenabgabe über die Revision des Heilmittelgesetzes abzuschaffen, geht in die gleiche Richtung: Ein patientenfreundliches, qualitätsgetriebenes und kostengünstiges Instrument bei der Gesundheitsversorgung soll der Bevölkerung in Zukunft nicht mehr zur Verfügung stehen. Mit solchen Fehlentscheiden fragt man sich schon zu Recht, wo einerseits die Bedürfnisse und der Mehrnutzen für Patienten, und andererseits die überall geforderte Stärkung der Grundversorgung und Hausarztmedizin bleiben.

Die Notwendigkeit der Revision der Tarifstruktur ist unbestritten, stammen doch die dem TARMED hinterlegten Kostendaten wie Mieten, Löhne des Praxispersonals, Gerätepreise usw. aus den Jahren 1994 bis 1996. Sie wurden seither nie angepasst. Die Preise des TARMED haben sich, wie das Bundesamt für Statistik im letzten Bulletin festhält, somit nicht verändert!

Aus verschiedenen Gründen wird die Revision seit Jahren von den Kostenträgern blockiert. Die FMH hat deshalb bereits im Sommer 2010 beschlossen, zusammen mit H+ die Revision der Tarifstruktur TARMED an die Hand zunehmen. Seit dem Kick-Off am 20. Oktober 2010 sind die Fachgesellschaften intensiv an der Arbeit. Ziel ist es, die Tarifstruktur auf das

\section{Die FMH und $\mathrm{H}+$ haben die TARMED- Revision bereits in Angriff genommen.}

Niveau 2011 zu bringen und sie für die kommenden Tarifverhandlungen fit zu machen. Das Projekt kommt gut voran. Erst nach der Genehmigung der Tarifstruktur durch TARMED Suisse können dann in einem zweiten Schritt auch Preisverhandlungen mit den Kostenträgern aufgenommen werden.

Verordnete Tariffestsetzungen des Bundes führen nicht zum Ziel! In unserem föderalistischen Gesundheitswesen können nur Verhandlungslösungen zum Ziel führen, das bestätigt auch das BAG in seinem Beibericht klar und deutlich. Dabei gilt es zu berücksichtigen, dass die ausgewogene Überarbeitung und Bewertung der einzelnen Tarifpositionen untereinander in der Revision der Tarifstruktur nicht mit der Preisbildung verknüpft wird. Die laufenden DRG-Verhandlungen zeigen dieses Vorgehen mit der Trennung von Tarifstruktur und Preisbildung exemplarisch auf.

Dr. med. Ernst Gähler, Vizepräsident der FMH, Verantwortlicher Ressort Tarife und Verträge 